See discussions, stats, and author profiles for this publication at: https://www.researchgate.net/publication/280498190

\title{
Analytic models for density of a ground-state spinor condensate
}

Article in Physical Review A · July 2015

DOI: 10.1103/PhysRevA.92.023616 - Source: arXiv

2 authors:

Sandeep Gautam

Indian Institute of Technology Ropar

32 PUBLICATIONS 232 CITATIONS

SEE PROFILE
Sadhan K. Adhikari

São Paulo State University

276 PUBLICATIONS 4,539 CITATIONS

SEE PROFILE 


\title{
Analytic models for density of a ground-state spinor condensate
}

\author{
Sandeep Gautam*1 and S. K. Adhikari ${ }^{\dagger 1}$ \\ ${ }^{1}$ Instituto de Física Teórica, Universidade Estadual Paulista - UNESP, \\ 01.140-070 São Paulo, São Paulo, Brazil
}

(Dated: July 29, 2015)

\begin{abstract}
We demonstrate that the ground state of a trapped spin-1 and spin-2 spinor ferromagnetic BoseEinstein condensate (BEC) can be well approximated by a single decoupled Gross-Pitaevskii (GP) equation. Useful analytic models for the ground-state densities of ferromagnetic BECs are obtained from the Thomas-Fermi approximation (TFA) to this decoupled equation. Similarly, for the ground states of spin-1 anti-ferromagnetic and spin-2 anti-ferromagnetic and cyclic BECs, some of the spin component densities are zero which reduces the coupled GP equation to a simple reduced form. Analytic models for ground state densities are also obtained for anti-ferromagnetic and cyclic BECs from the TFA to the respective reduced GP equations. The analytic densities are illustrated and compared with the full numerical solution of the GP equation with realistic experimental parameters.
\end{abstract}

PACS numbers: 03.75.Mn, 03.75.Hh, 67.85.Bc, 67.85.Fg

\section{INTRODUCTION}

The advent of the optical traps paved the way for the first realization of a Bose-Einstein condensate (BEC) with internal spin degrees of freedom 1], also known as a spinor BEC. Since then, a lot of theoretical and experimental studies have been performed on the spinor BECs 2 4. In contrast to a scalar BEC, which is characterized by a single interaction parameter, the spin1 [5] and spin-2 [6, 7] BECs have, respectively, two and three interaction parameters. Depending on the relative strength of the interaction parameters, a spin1 BEC in the absence of external magnetic field can be either in a ferromagnetic or an anti-ferromagnetic phase [5]. In the presence of magnetic field the ground state phase diagram of spin-1 condensate has been investigated both for uniform [8]10, and trapped systems [10, 11]. Similarly, in the absence of external magnetic field, a spin-2 BECs can be in one of the possible three ground state phases: ferromagnetic, anti-ferromagnetic and cyclic [6, 7]. The spin-1 and spin-2 BECs are described by three- and five-component complex order parameters, respectively, thus leading to coupled meanfield Gross-Pitaevskii (GP) equations involving threeand five-component wave functions, which, unlike in a scalar BEC, could be complex in general. A numerical solution of these equations could be cumbersome for both spin-1 12, 13] and spin-2 [14, 15] BECs. In this paper, we propose simple and useful analytic models for the densities of ground states of quasi-one-dimensional (quasi1D), circularly-symmetric quasi-two-dimensional (quasi2D) [16], and spherically-symmetric three-dimensional (3D) spin-1 and spin-2 spinor BECs. Here, we consider nearly-overlapping spatially-symmetric ground states only. Phase-separated spatially-asymmetric pro-

*sandeepgautam24@gmail.com ${ }_{\dagger}$ adhikari44@yahoo.com, URL http://www.ift.unesp.br/users/adhikari than the oscillator length $l_{0}$, i.e. $R / l_{0}>>1$ [18, 19 . The files [13] do not appear as ground states and will not be considered.

The two interaction parameters for a spin-1 BEC are $c_{0} \propto\left(a_{0}+2 a_{2}\right) / 3$ and $c_{1} \propto\left(a_{2}-a_{0}\right) / 3[5]$, whereas the three interaction parameters for a spin-2 BEC are $c_{0} \propto\left(4 a_{2}+3 a_{4}\right) / 7, c_{1} \propto\left(a_{4}-a_{2}\right) / 7$, and $c_{2} \propto\left(7 a_{0}-\right.$ $\left.10 a_{2}+3 a_{4}\right) / 7$ [6, 7], where $a_{0}, a_{2}$, and $a_{4}$ are $s$-wave scattering lengths in total spin $f_{\text {tot }}=0,2$, and 4 channels, respectively. For a ferromagnetic BEC, e.g. for $c_{1}<0$ for a spin-1 BEC, and for $c_{1}<0$ and $c_{2}>20 c_{1}$ for a spin-2 BEC, we find that to a very good approximation the densities for different spin components $m_{f}$ of the ground-state wave function with magnetization $m$ are multiples of each other. This allows one to replace the coupled GP equation for the ground-state wave function by a single partial differential equation, which we call the decoupled-mode (DM) equation. On the other hand, for an anti-ferromagnetic BEC, e.g. for $c_{1}>0$ for a spin-1 $\mathrm{BEC}$, and for $c_{2}<0$ and $c_{2}<20 c_{1}$ for a spin-2 BEC, we find that the densities, for some of the spin components, of the ground-state wave function with magnetization $m$ are identically zero, thus reducing the original coupled GP equation to a system of two coupled equations for any non-zero magnetization. Similarly, for a cyclic BEC, e.g. for $c_{1}>0$ and $c_{2}>0$ for a spin-2 BEC, the fivecomponent GP equation reduces to a system of two or three coupled equations. These reduced GP equations and the DM equation for the ground state of a spinor BEC in different parameter domains, valid in all spatial dimensions, are solved in the Thomas-Fermi approximation (TFA) (or local-density approximation) to yield simple analytic models for the ground-state densities of spin-1 and spin-2 spinor BECs.

The TFA is applicable when the interaction energy in the GP equation is much larger than the kinetic energy term, so that the latter could be neglected, thus leading to simple analytic formulae for the condensate densities [17. In a repulsive scalar BEC, applicability of TFA requires that the size of the condensate $R$ is much larger 
spatial extent of the BEC in units of $l_{0}$ is

$$
l_{\mathcal{D}}=\frac{R}{l_{0}} \sim\left(\frac{N a}{l_{0}}\right)^{1 /(\mathcal{D}+2)}
$$

where $\mathcal{D}=1,2,3$ is the dimensionality of the space [18, 19]. The criterion is satisfied if the dimensionless parameter $N a / l_{0}>>1$. The ratio $N a / l_{0}$ is a measure of the strength of repulsive interaction. For a spinor BEC, the applicability of TFA for $m_{f}$ component requires that its spatial extent is much larger than $l_{0}$.

There have been few studies to include the neglected kinetic energy contribution in the TFA [18, 20]. Earlier, the TFA was used to study the ground state properties of binary condensates 21] and spin dynamics in quasi-1D spin-1 condensate [22]. Spin-orbit-coupled pseudospin$1 / 2$ BECs under rotation have also been theoretically investigated using the TFA [23].

We use the experimentally realizable trapping potential and interaction parameters to illustrate the present analytic models for ground-state densities in different parameter domains. In the case of a spin-1 BEC, the background scattering lengths of ${ }^{87} \mathrm{Rb}$ and ${ }^{23} \mathrm{Na}$ fall in the ferromagnetic [24, 25] and anti-ferromagnetic [26] domains, respectively, and we use these to study the ground state properties. In the case of a spin-2 BEC, we employ ${ }^{23} \mathrm{Na}$ and ${ }^{83} \mathrm{Rb}$ BECs for the illustration. The background scattering lengths of spin- $2{ }^{23} \mathrm{Na}$ and ${ }^{83} \mathrm{Rb}$ correspond to the anti-ferromagnetic and ferromagnetic phases, respectively 6]. By tuning one of the scattering lengths of ${ }^{23} \mathrm{Na}$, one can move from anti-ferromagnetic to either ferromagnetic or cyclic phase. Experimentally, such a change of single scattering length can be achieved by exploiting magnetic 27] and optical 28] Feshbach resonance techniques. The results of the analytic models are also validated by a numerical solution of the original mean-field GP equations for quasi-1D and quasi-2D traps.

In Sec. II], we present the full mean-field GP equations for spin-1 and spin-2 BECs and derive the reduced meanfield GP equations for the ground-state wave function in the different parameter domains. By assuming that the component wavefunctions of a ferromagnetic BEC are proportional to each other, which is indeed the case as suggested by numerical simulations, we derive the DM equation. By minimizing the $c_{1}$ - and $c_{2}$-dependent energy terms for the ground-state wave function, we obtain the reduced GP equations in all parameter domains. In Secs. III and IV we obtain the analytic models for spin-1 and spin-2 ground-state BECs, respectively, by employing the TFA to the reduced GP and the DM equations. A comparison of the analytic densities with the numerical densities obtained from the full GP equations leads to a very satisfactory agreement. In Sec. V] we present a summary and concluding remarks. Some of the technical details about the derivation of the DM equation and the reduced GP equations in different parameter domains are presented in Appendix A and B.

\section{REDUCED MEAN-FIELD EQUATIONS}

\section{A. Spin-1 BEC}

The coupled GP equations for different spin components $m_{f}= \pm 1,0$, for a spin- 1 BEC of $N$ atoms of mass $M$ each can be written in dimensionless form as [2]

$$
\begin{aligned}
\mu_{ \pm 1} \phi_{ \pm 1}(\mathbf{x}) & =\mathcal{H} \phi_{ \pm 1}(\mathbf{x})+c_{0} \rho \phi_{ \pm 1}(\mathbf{x}) \pm c_{1} F_{z} \phi_{ \pm 1}(\mathbf{x}) \\
& +\left(c_{1} / \sqrt{2}\right) F_{\mp} \phi_{0}(\mathbf{x}) \\
\mu_{0} \phi_{0}(\mathbf{x}) & =\mathcal{H} \phi_{0}(\mathbf{x})+c_{0} \rho \phi_{0}(\mathbf{x})+\left(c_{1} / \sqrt{2}\right)\left[F_{-} \phi_{-1}(\mathbf{x})\right. \\
& \left.+F_{+} \phi_{+1}(\mathbf{x})\right]
\end{aligned}
$$

where

$$
\begin{aligned}
& F_{ \pm} \equiv F_{x} \pm F_{y}=\sqrt{2}\left[\phi_{ \pm 1}^{*}(\mathbf{x}) \phi_{0}(\mathbf{x})+\phi_{0}^{*}(\mathbf{x}) \phi_{\mp 1}(\mathbf{x})\right] \\
& F_{z}=\rho_{+1}(\mathbf{x})-\rho_{-1}(\mathbf{x}), \quad \mathcal{H}=\left[-\frac{1}{2} \nabla^{2}+V(\mathbf{x})\right]
\end{aligned}
$$

and the component density $\rho_{j}=\left|\phi_{j}(\mathbf{x})\right|^{2}$ with $j= \pm 1,0$, the total density $\rho=\sum_{j} \rho_{j}$, and $\mu_{ \pm 1}, \mu_{0}$ are the respective chemical potentials and ${ }^{*}$ denotes complex conjugate. In $3 \mathrm{D}$, the interaction parameters, Laplacian, and trapping potential are defined as

$$
\begin{aligned}
c_{0} & =\frac{4 \pi N\left(a_{0}+2 a_{2}\right)}{3 l_{0}}, c_{1}=\frac{4 \pi N\left(a_{2}-a_{0}\right)}{3 l_{0}}, \\
\nabla^{2} & =\frac{\partial^{2}}{\partial x^{2}}+\frac{\partial^{2}}{\partial y^{2}}+\frac{\partial^{2}}{\partial z^{2}}, V(\mathbf{x})=\frac{x^{2}+\beta^{2} y^{2}+\gamma^{2} z^{2}}{2},
\end{aligned}
$$

with $\mathbf{x} \equiv\{x, y, z\} . \quad$ Here $l_{0}=\sqrt{\hbar /\left(M \omega_{x}\right)}, \beta=\omega_{y} / \omega_{x}$, $\gamma=\omega_{z} / \omega_{x}$, where $\omega_{x}, \omega_{y}, \omega_{z}$ are the confining trap frequencies in $x, y, z$ directions, respectively. When the trapping frequency along one axis, say $\omega_{z}$, is much larger than the geometric mean of other two, i.e., $\omega_{z} \gg \sqrt{\omega_{x} \omega_{y}}$, then one can approximate the Eqs. (2) - (3) by quasi two-dimensional (2D) equations which can be obtained by substituting 16 .

$$
\begin{aligned}
c_{0} & =\frac{2 N \sqrt{2 \pi}\left(a_{0}+2 a_{2}\right)}{3 l_{z}}, c_{1}=\frac{2 N \sqrt{2 \pi}\left(a_{2}-a_{0}\right)}{3 l_{z}}, \\
\nabla^{2} & =\frac{\partial^{2}}{\partial x^{2}}+\frac{\partial^{2}}{\partial y^{2}}, V(\mathbf{x})=\frac{x^{2}+\beta^{2} y^{2}}{2}, \mathbf{x} \equiv\{x, y\},
\end{aligned}
$$

in Eqs. $22-(3)$, here $l_{z}=\sqrt{\hbar /\left(M \omega_{z}\right)}$. Similarly, if the trapping frequencies along two axes, say $y$ and $z$, are much larger than the third frequency $\omega_{x}$, Eqs. (2) - (3) can be approximated by quasi-1D equations which can be obtained by substituting

$$
\begin{aligned}
c_{0} & =\frac{2 N\left(a_{0}+2 a_{2}\right) l_{0}}{3 l_{y z}^{2}}, c_{1}=\frac{2 N\left(a_{2}-a_{0}\right) l_{0}}{3 l_{y z}^{2}} \\
\nabla^{2} & =\frac{\partial^{2}}{\partial x^{2}}, V(\mathbf{x})=\frac{x^{2}}{2}, \mathbf{x} \equiv x
\end{aligned}
$$

where $l_{y z}=\sqrt{\hbar /\left(M \omega_{y z}\right)}$ and $\omega_{y z}=\sqrt{\omega_{y} \omega_{z}}$. Here length is measured in units of $l_{0}$, density in units of $l_{0}^{-\mathcal{D}}$ and 
chemical potential in units of $\hbar \omega_{x}$, where $\mathcal{D}=1,2,3$ is the dimensionality of space. The total density is normalized to unity $\int d \mathbf{x} \rho(\mathbf{x})=1$. The volume element $d \mathbf{x}=2 d X$ in $1 \mathrm{D}, 2 \pi X d X$ in $2 \mathrm{D}$ with circular symmetry, and $4 \pi X^{2} d X$ in $3 \mathrm{D}$ with spherical symmetry, where $X=|\mathbf{x}|$ is the length of the vector $\mathbf{x}$. In this paper, we consider isotropic $3 \mathrm{D}$ and isotropic quasi-2D traps, i.e., $\beta=\gamma=1$ for $3 \mathrm{D}$ traps and $\beta=1 \ll \gamma$ for quasi-2D traps.

Numerical calculation for the ground-state densities of a of a ferromagnetic BEC $\left(c_{1}<0\right)$ has revealed that the component densities are essentially multiples of each other. This opens the possibility of writing a single decoupled mode (DM) equation for the wave-function $\phi_{\mathrm{DM}}$ for the ferromagnetic BEC and obtain the component wave functions as multiples of this wave function according to

$$
\phi_{j}(\mathbf{x})=\alpha_{j} \phi_{\mathrm{DM}}(\mathbf{x}), \quad j= \pm 1,0,
$$

where $\alpha_{j}$ 's, in general, are complex numbers. The conditions (12) when substituted in Eqs. (2) and (3) lead to three different equations for the same wave function $\phi_{\mathrm{DM}}$. A consistency requirement on these three equations leads to the single decoupled-mode (DM) equation for the wave function $\phi_{\mathrm{DM}}$ :

$$
\mu \phi_{\mathrm{DM}}(\mathbf{x})=\left[-\frac{1}{2} \nabla^{2}+V(\mathbf{x})+C \phi_{\mathrm{DM}}^{2}(\mathbf{x})\right] \phi_{\mathrm{DM}}(\mathbf{x})
$$

with $C \equiv \mathcal{C}_{I}=c_{0}+c_{1}$ and normalization $\int \rho_{\mathrm{DM}}(\mathbf{x}) d \mathbf{x}=$ 1 , provided that

$$
\begin{aligned}
\left|\alpha_{ \pm 1}\right| & =\frac{1 \pm m}{2}, \quad\left|\alpha_{0}\right|=\frac{\sqrt{1-m^{2}}}{\sqrt{2}} \\
m & \equiv \int d \mathbf{x}\left[\rho_{+1}-\rho_{-1}\right]=\left|\alpha_{+1}\right|^{2}-\left|\alpha_{-1}\right|^{2}
\end{aligned}
$$

where $m$ is the magnetization. An equation similar to Eq. (13) with $C=c_{0} \sim\left(a_{0}+2 a_{2}\right) / 3$, known as the singlemode approximation (SMA) 29], was obtained before as an approximation to Eqs. (2)-(3). The component densities were then obtained using Eq. (14). In the DM model we have a different $C \equiv \mathcal{C}_{I}=\left(c_{0}+c_{1}\right) \sim a_{2}$, which is independent of $a_{0}$. Equation (13) was previously obtained by Yi et al. 30, as an improvement over the SMA. The breakdown of the single-mode approximation for trapped spin-1 condensates in the presence of magnetic field has also been theoretically investigated [10].

Provided that ansatz (12) holds, distribution (14) can be obtained independently from a consideration of $c_{1^{-}}$ dependent energy minimization for a ferromagnetic BEC as shown in Appendix A. The DM equation is very useful for finding the ground state of a ferromagnetic BEC where all density components are non-zero and this procedure can also be readily generalized to higher-spin cases as shown in Sec. IIB for a spin-2 ferromagnetic BEC.

For the ground-state of an anti-ferromagnetic BEC $\left(c_{1}>0\right)$ with non-zero magnetization, energy minimization requires that $\phi_{0}(\mathbf{x})=0$, viz. Appendix A. Then the normalization and magnetization conditions yield

$$
\int \rho_{ \pm 1} d \mathbf{x}=\frac{1 \pm m}{2}, \quad \rho_{0}=0
$$

For $m=0$, besides the aforementioned state, there is another degenerate state where all the atoms are in $m_{f}=0$ component, i.e. $\rho_{ \pm 1}=0$ and $\int \rho_{0} d \mathbf{x}=1$. Unlike in a ferromagnetic BEC, ansatz $\sqrt{12}$ does not hold for an anti-ferromagnetic BEC for a non-zero magnetization $m$ where different components occupy different spatial extensions. On the other hand, for $m=0$, SMA becomes exact in this phase [30, as the $c_{1}$-dependent term vanishes.

We will derive the analytic model for a ferromagnetic BEC using the TFA to the DM equation (13), whereas for an anti-ferromagnetic BEC we rely on the TFA to the GP equation (2) with $\phi_{0}(\mathbf{x})=0$ for the same.

\section{B. Spin-2 BEC}

The dimensionless coupled GP equations for different spin components $m_{f}= \pm 2, \pm 1,0$, for a spin- 2 BEC can be written as [2]

$$
\begin{aligned}
& \mu_{ \pm 2} \phi_{ \pm 2}(\mathbf{x})=\mathcal{H} \phi_{ \pm 2}(\mathbf{x})+c_{0} \rho \phi_{ \pm 2}(\mathbf{x})+\left(c_{2} / \sqrt{5}\right) \Theta \phi_{\mp 2}^{*}(\mathbf{x}) \\
& \quad+c_{1}\left[F_{\mp} \phi_{ \pm 1}(\mathbf{x}) \pm 2 F_{z} \phi_{ \pm 2}(\mathbf{x})\right], \\
& \mu_{ \pm 1} \phi_{ \pm 1}(\mathbf{x})=\mathcal{H} \phi_{ \pm 1}(\mathbf{x})+c_{0} \rho \phi_{ \pm 1}(\mathbf{x})-\left(c_{2} / \sqrt{5}\right) \Theta \phi_{\mp 1}^{*}(\mathbf{x}) \\
& \quad+c_{1}\left[\sqrt{3 / 2} F_{\mp} \phi_{0}(\mathbf{x})+F_{ \pm} \phi_{ \pm 2}(\mathbf{x}) \pm F_{z} \phi_{ \pm 1}(\mathbf{x})\right] \\
& \mu_{0} \phi_{0}(\mathbf{x})=\mathcal{H} \phi_{0}(\mathbf{x})+c_{0} \rho \phi_{0}(\mathbf{x})+\left(c_{2} / \sqrt{5}\right) \Theta \phi_{0}^{*}(\mathbf{x}) \\
& \quad+c_{1} \sqrt{3 / 2}\left[F_{-} \phi_{-1}(\mathbf{x})+F_{+} \phi_{+1}(\mathbf{x})\right],
\end{aligned}
$$

where

$$
\begin{aligned}
F_{+}= & F_{-}^{*}=2\left(\phi_{+2}^{*} \phi_{+1}+\phi_{-1}^{*} \phi_{-2}\right) \\
& +\sqrt{6}\left(\phi_{+1}^{*} \phi_{0}+\phi_{0}^{*} \phi_{-1}\right), \\
F_{z}= & 2\left(\left|\phi_{+2}\right|^{2}-\left|\phi_{-2}\right|^{2}\right)+\left|\phi_{+1}\right|^{2}-\left|\phi_{-1}\right|^{2}, \\
\Theta= & \frac{2 \phi_{+2} \phi_{-2}-2 \phi_{+1} \phi_{-1}+\phi_{0}^{2}}{\sqrt{5}} .
\end{aligned}
$$

Here the interaction parameters $c_{0}=4 \pi N\left(4 a_{2}+\right.$ $\left.3 a_{4}\right) /\left(7 l_{0}\right), c_{1}=4 \pi N\left(a_{4}-a_{2}\right) /\left(7 l_{0}\right), c_{2}=4 \pi N\left(7 a_{0}-\right.$ $\left.10 a_{2}+3 a_{4}\right) /\left(7 l_{0}\right), \mu_{ \pm 2}, \mu_{ \pm 1}$, and $\mu_{0}$ are the respective chemical potentials. All repeated variables have the same meaning as in the spin- 1 case. The total density $\rho$ is again normalized to unity. As in the spin-1 case, GP equations in quasi-2D traps can be obtained by using Eqs. (9) and substituting $c_{0}=2 N \sqrt{2 \pi}\left(4 a_{2}+3 a_{4}\right) /\left(7 l_{z}\right), c_{1}=$ $2 N \sqrt{2 \pi}\left(a_{4}-a_{2}\right) /\left(7 l_{z}\right), c_{2}=2 N \sqrt{2 \pi}\left(7 a_{0}-10 a_{2}+\right.$ $\left.3 a_{4}\right) /\left(7 l_{z}\right)$ in Eqs. (17)-(19). Similarly, GP equations in quasi-1D traps can be obtained by using Eqs. (11) and substituting $c_{0}=2 N\left(4 a_{2}+3 a_{4}\right) l_{0} /\left(7 l_{y z}^{2}\right), c_{1}=2 N\left(a_{4}-\right.$ $\left.a_{2}\right) l_{0} /\left(7 l_{y z}^{2}\right), c_{2}=2 N\left(7 a_{0}-10 a_{2}+3 a_{4}\right) l_{0} /\left(7 l_{y z}^{2}\right)$ in Eqs. (17) $-(19)$. 
In the DM, for a ferromagnetic BEC $\left(c_{1}<0, c_{2}>\right.$ $20 c_{1}$ ) with all non-zero component densities, if we substitute the ansatz

$$
\phi_{j}=\alpha_{j} \phi_{\mathrm{DM}}, \quad j= \pm 2, \pm 1,0,
$$

in Eqs. 17), (18), and (19), we obtain five independent equations for $\phi_{\mathrm{DM}}$. A consistency requirement among these five equations for the $c_{1}$-dependent terms leads to the DM equation with $C \equiv \mathcal{C}_{I I}=\left(c_{0}+4 c_{1}\right)$, provided that

$$
\begin{aligned}
\left|\alpha_{ \pm 2}\right| & =\frac{(2 \pm m)^{2}}{16} \\
\left|\alpha_{ \pm 1}\right| & =\frac{\sqrt{4-m^{2}}(2 \pm m)}{8} \\
\left|\alpha_{0}\right| & =\frac{1}{8} \sqrt{\frac{3}{2}}\left(4-m^{2}\right)
\end{aligned}
$$

with magnetization and normalization conditions

$$
\begin{aligned}
m & \equiv \int d \mathbf{x}\left[2\left(\rho_{+2}-\rho_{-2}\right)+\left(\rho_{+1}-\rho_{-1}\right)\right] \\
& =2\left(\left|\alpha_{+2}\right|^{2}-\left|\alpha_{-2}\right|^{2}\right)+\left(\left|\alpha_{+1}\right|^{2}-\left|\alpha_{-1}\right|^{2}\right) \\
1 & =\left|\alpha_{+2}\right|^{2}+\left|\alpha_{-2}\right|^{2}+\left|\alpha_{+1}\right|^{2}+\left|\alpha_{-1}\right|^{2}+\left|\alpha_{0}\right|^{2}
\end{aligned}
$$

In the DM model for a spin-2 BEC $C \equiv \mathcal{C}_{I I} \sim a_{4}$ is independent of the scattering lengths $a_{0}$ and $a_{2}$, with $a_{4}$ playing the role of scattering length in an equivalent scalar BEC. With the coefficients $\alpha_{ \pm 2}, \alpha_{ \pm 1}, \alpha_{0}$ given by Eqs. (24)- 26) the coefficient $\Theta$ of Eq. 22 is identically equal to 0 . The condition $\Theta=0$ for the ground state makes the GP equations (17)- 19 simpler and independent of $c_{2}$. Consequently, the DM equation $(13)$ becomes an exact equation for the ground state wave function provided Eq. 23 holds, e.g. the component wave functions are multiples of each other. Our numerical calculations show that the condition 23 holds for all magnetization to a very high degree of accuracy.

The coefficients $\alpha_{ \pm 2}, \alpha_{ \pm 1}, \alpha_{0}$ can also be obtained from a minimization of energy along with condition (23), for a ferromagnetic ground state with $c_{1}<0$ and $c_{2}>20 c_{1}$. An explicit account of the derivation of the coefficients $\alpha_{ \pm 2}, \alpha_{ \pm 1}, \alpha_{0}$, from an energy minimization for a ferromagnetic spin-2 BEC is given in Appendix B.

For an anti-ferromagnetic BEC $\left(c_{2}<0, c_{2}<20 c_{1}\right)$ for any non-zero magnetization $m$ numerical studies show that $\phi_{ \pm 1}(\mathbf{x})=\phi_{0}(\mathbf{x})=0$ for the ground state. This can also be obtained from energetic consideration as shown in Appendix B. The magnetization and normalization conditions 27) and (29) then yield

$$
\int \rho_{ \pm 2} d \mathbf{x}=\frac{2 \pm m}{4}
$$

Energy consideration establishes that a cyclic BEC $\left(c_{1}>\right.$ $\left.0, c_{2}>0\right)$ has two degenerate ground states for all non-zero magnetization $m$ with $(i) \phi_{+1}(\mathbf{x})=\phi_{0}(\mathbf{x})=$ $\phi_{-2}(\mathbf{x})=0$, or with $(i i) \phi_{ \pm 1}(\mathbf{x})=0$, viz. Appendix B.

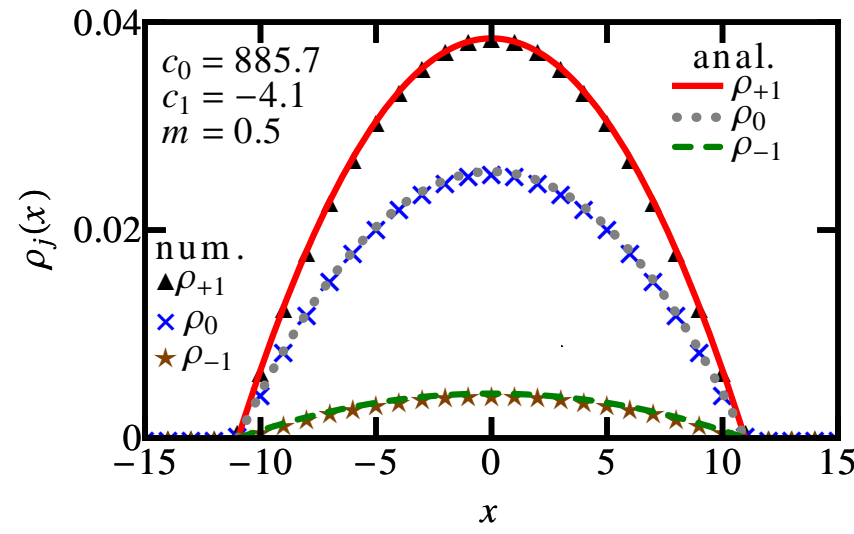

FIG. 1: (Color online) Analytic (anal.) and numerical (num.) densities of a spin-1 quasi-1D ferromagnetic ${ }^{87} \mathrm{Rb}$ BEC. The number of atoms, scattering lengths and oscillator lengths are, respectively, $N=10,000, a_{0}=101.8 a_{B}, a_{2}=100.4 a_{B}$ [24], $l_{0}=2.41 \mu \mathrm{m}, l_{y z}=0.54 \mu \mathrm{m}$, here $a_{B}$ is Bohr radius.

Consequently, the magnetization and normalization conditions (27) and 29) lead for these two states

(i) $\int d \mathbf{x} \rho_{+2}=\frac{1+m}{3}, \quad \int d \mathbf{x} \rho_{-1}=\frac{2-m}{3}$,

(ii) $\int d \mathbf{x} \rho_{ \pm 2}=\left(\frac{2 \pm m}{4}\right)^{2}, \quad \int d \mathbf{x} \rho_{0}=\frac{4-m^{2}}{8}$.

For both anti-ferromagnetic and cyclic BECs we will derive the analytic models directly from the TFA to the GP equations (17)-(19) and not from the DM, whereas for a ferromagnetic $\overline{\mathrm{BEC}}$ we will rely on the TFA to the DM equation with $C=\left(c_{0}+4 c_{1}\right)$.

\section{ANALYTIC MODEL FOR SPIN-1 BEC}

\section{A. Ferromagnetic BEC}

We derive the analytic model for the ground-state density of a spin-1 BEC using the TFA to the DM equation (13) with component densities given by Eq. (14). In the TFA the kinetic energy term in Eq. 113 is neglected, which is reasonable for a moderate to large positive nonlinear terms, and the BEC density is calculated by equating the "Hamiltonian" to the chemical potential by

$$
\mu=\left[X^{2} / 2+C \rho_{\mathrm{DM}}\right], \quad C \equiv \mathcal{C}_{I}=c_{0}+c_{1},
$$

thus leading to the TFA density

$$
\rho_{\mathrm{DM}}(X)=\left(l_{\mathcal{D}}^{2}-X^{2}\right) /\left(2 \mathcal{C}_{I}\right), \quad X \leq l_{\mathcal{D}}=\sqrt{2 \mu} .
$$

Imposing the condition of normalization $\int \rho_{\mathrm{DM}}(X) d \mathbf{x}=$ 1 , we obtain, in $1 \mathrm{D}, 2 \mathrm{D}$, and $3 \mathrm{D}$, respectively $l_{\mathcal{D}}=$ $\left(3 \mathcal{C}_{I} / 2\right)^{1 / 3},\left(4 \mathcal{C}_{I} / \pi\right)^{1 / 4}$ and $\left(15 \mathcal{C}_{I} / 4 \pi\right)^{1 / 5}$, provided $\mathcal{C}_{I}>$ 


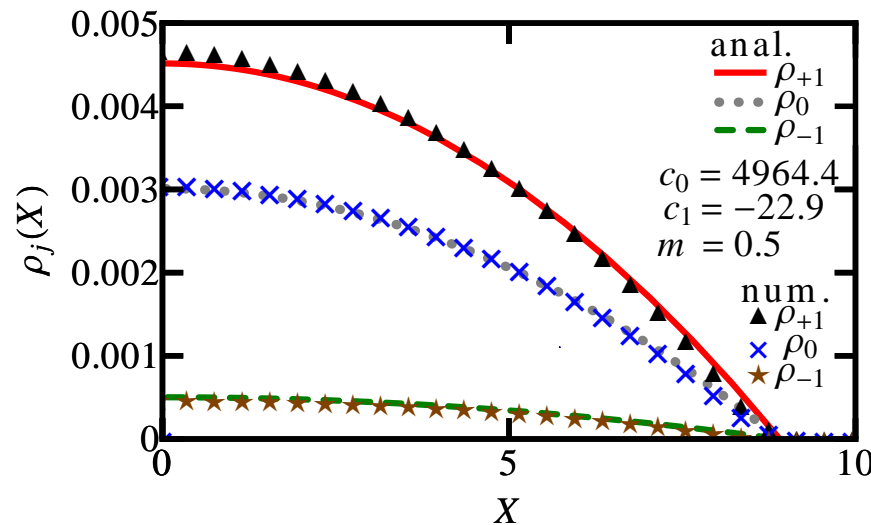

FIG. 2: (Color online) Analytic (anal.) and numerical (num.) densities of a spin-1 quasi-2D ferromagnetic ${ }^{87} \mathrm{Rb}$ BEC. The number of atoms, scattering lengths and oscillator lengths are, respectively, $N=100,000, a_{0}=101.8 a_{B}, a_{2}=100.4 a_{B}$ [24], $l_{0}=2.41 \mu \mathrm{m}, l_{z}=0.54 \mu \mathrm{m}$, here $a_{B}$ is Bohr radius.

0 . The component densities are calculated using Eqs. (12) and (14). The analytic densities for a quasi-1D spin1 ferromagnetic ${ }^{87} \mathrm{Rb} \mathrm{BEC}$ are shown in Fig. 1 along with the numerical solution of the full coupled GP equations (2)-(3). The same for a quasi-2D spin-1 ferromagnetic ${ }^{87} \mathrm{Rb} \mathrm{BEC}$ is shown in Fig. 2 All numerical calculations are performed using the split-step Crank-Nicolson scheme [31] with space and time steps 0.025 and 0.00005 , respectively.

In the DM model $\mathcal{C}_{I} \sim a_{2}$ plays the same role as the scattering length $a$ in a scalar BEC in Eq. (1). Hence in this case the condition of validity of the TFA will be $N a_{2} / l_{0}>>1$

\section{B. Anti-ferromagnetic BEC}

In this case, the analytic model is derived by applying TFA directly to the GP equation (2) with $\phi_{0}(\mathbf{x})=0$. For a non-zero magnetization $(0<m<1)$, the $m_{f}=+1$ component accommodates more atoms and its spatial extension $l_{\mathcal{D}(+1)}$ is larger than the same of the the $m_{f}=-1$ component with spatial extension $l_{\mathcal{D}(-1)}$. Hence for $l_{\mathcal{D}(+1)}>x>l_{\mathcal{D}(-1)}, \phi_{-1}(\mathbf{x})=0$ and the coupled GP equation (2) for $\phi_{ \pm 1}(\mathbf{x})$ reduces to a single equation for $\phi_{+1}(\mathbf{x})$. In the TFA, the kinetic energy terms in the GP equation (2) are neglected and the densities are calculated by equating the Hamiltonian to the respective chemical potentials:

$$
\begin{aligned}
& \mu_{ \pm 1}=\left[X^{2} / 2+c_{0} \rho\right]+c_{1}\left(\rho_{ \pm 1}-\rho_{\mp 1}\right), \quad X \leq l_{\mathcal{D}(-1)}, \\
& \mu_{+1}=\left[X^{2} / 2+\mathcal{C}_{I} \rho_{+1}\right], \quad l_{\mathcal{D}(+1)} \geq X \geq l_{\mathcal{D}(-1)},
\end{aligned}
$$

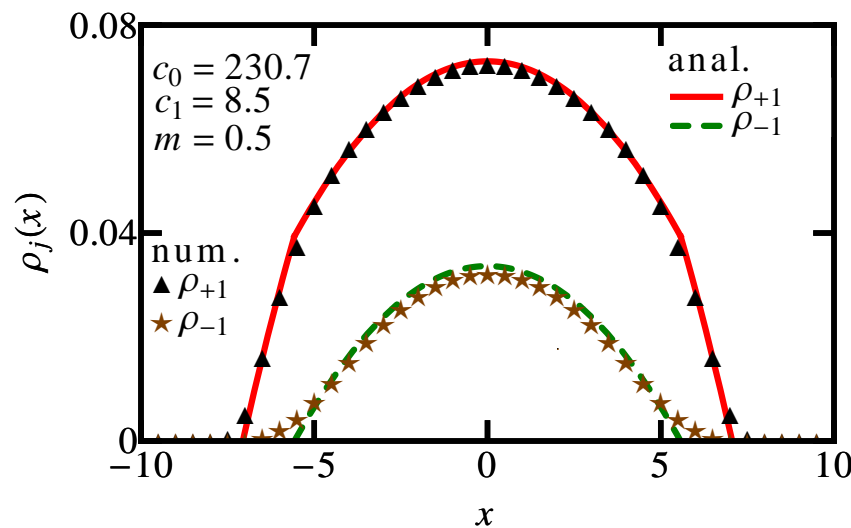

FIG. 3: (Color online) Analytic (anal.) and numerical (num.) densities of a spin-1 quasi-1D anti-ferromagnetic ${ }^{23} \mathrm{Na}$ BEC. The number of atoms, scattering lengths and oscillator lengths are, respectively, $N=10,000 ; a_{0}=47.36 a_{B}$, $a_{2}=52.98 a_{B}\left[\underline{3} ; l_{0}=4.69 \mu \mathrm{m}, l_{y z}=1.05 \mu \mathrm{m}\right.$.

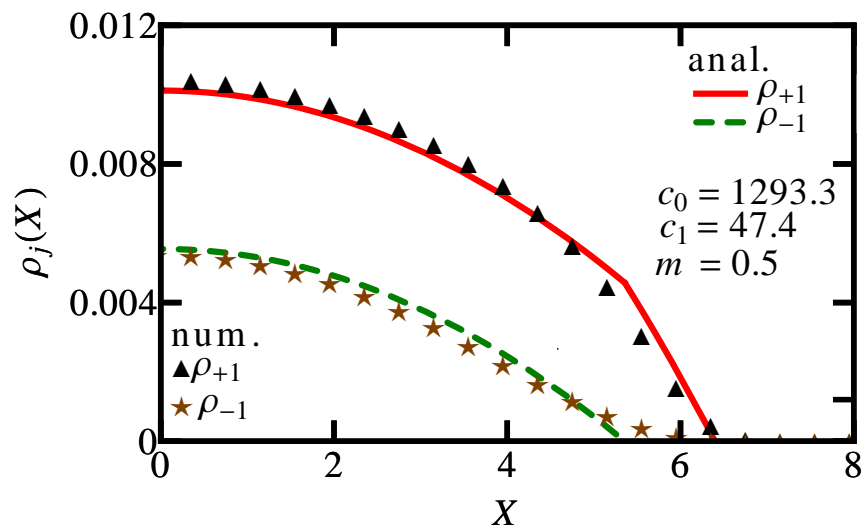

FIG. 4: (Color online) Analytic (anal.) and numerical (num.) densities of a spin-1 quasi-2D anti-ferromagnetic ${ }^{23} \mathrm{Na}$ BEC. The number of atoms, scattering lengths and oscillator lengths are, respectively, $N=100,000 ; a_{0}=47.36 a_{B}$, $a_{2}=52.98 a_{B}[3] ; l_{0}=4.69 \mu \mathrm{m}, l_{z}=1.05 \mu \mathrm{m}$.

subject to normalization 16. In the domain $l_{\mathcal{D}(+1)} \geq X \geq l_{\mathcal{D}(-1)}$, Eq. 36 has the solution

$$
\begin{aligned}
\rho_{+1}(X) & =\frac{l_{\mathcal{D}(+1)}^{2}-X^{2}}{2 \mathcal{C}_{I}}, \quad l_{\mathcal{D}(+1)} \geq X \geq l_{\mathcal{D}(-1)}, \\
\mu_{+1} & =l_{\mathcal{D}(+1)}^{2} / 2 .
\end{aligned}
$$

In the overlap region $X \leq l_{\mathcal{D}(-1)}$, coupled equations 35 have the solution

$$
\rho_{ \pm 1}(X)=\frac{c_{0}\left(\mu_{ \pm 1}-\mu_{\mp 1}\right)+c_{1}\left(\mu_{+1}+\mu_{-1}-X^{2}\right)}{4 c_{0} c_{1}} .
$$


The condition $\rho_{-1}\left(l_{\mathcal{D}(-1)}\right)=0$ leads to

$$
\mu_{-1}=\frac{\left(c_{0}-c_{1}\right) l_{\mathcal{D}(+1)}^{2}+2 c_{1} l_{\mathcal{D}(-1)}^{2}}{2 \mathcal{C}_{I}}
$$

Substituting Eqs. (38) and 40 in Eq. 39, we obtain

$$
\begin{gathered}
\rho_{+1}(X)=\frac{2 c_{0} l_{\mathcal{D}(+1)}^{2}+\left(c_{1}-c_{0}\right) l_{\mathcal{D}(-1)}^{2}-\mathcal{C}_{I} X^{2}}{4 c_{0} \mathcal{C}_{I}}, \quad X \leq l_{\mathcal{D}(-1)} \\
\rho_{-1}(X)=\frac{\left(l_{\mathcal{D}(-1)}^{2}-X^{2}\right)}{4 c_{0}}, \quad X \leq l_{\mathcal{D}(-1)} .
\end{gathered}
$$

The normalization condition 16 for $\rho_{ \pm 1}(X)$ leads to

$$
\begin{aligned}
& l_{\mathcal{D}(-1)}=l_{\mathcal{D}}\left[c_{0}(1-m) / \mathcal{C}_{I}\right]^{1 /(2+\mathcal{D})} \\
& l_{\mathcal{D}(+1)}=l_{\mathcal{D}}\left[\left(c_{0}+c_{1} m\right) / \mathcal{C}_{I}\right]^{1 /(2+\mathcal{D})}
\end{aligned}
$$

The densities (37), (41) and (42) with extensions given by Eqs. (43)- (44) constitute the analytic model in this case.

These analytic densities for a spin-1 quasi-1D antiferromagnetic ${ }^{23} \mathrm{Na}$ BEC are shown in Fig. 3 along with the numerical solution of the full coupled GP equations (2)-(3). The same for a spin-1 quasi-2D antiferromagnetic ${ }^{23} \mathrm{Na} \mathrm{BEC}$ are shown in Fig. 4. Comparing Eq. (1) with Eqs. (43)-(44), the conditions for the validity of TFA in this case are

$$
\begin{aligned}
\frac{N\left(a_{0}+2 a_{2}\right)(1-m)}{3 l_{0}} & >1, \\
\frac{N\left[a_{0}+2 a_{2}+\left(a_{2}-a_{0}\right) m\right]}{3 l_{0}} & >>1,
\end{aligned}
$$

for $m_{f}=-1$ and $m_{f}=+1$ component, respectively. The terms on the left side of Eqs. (45) and (46) are the measure of the repulsive interactions in $m_{f}=-1$ and $m_{f}=+1$ components, respectively.

For magnetization $m=0$ there is another degenerate ground state where all atoms are in the $m_{f}=0$ component [2]. In that case the spin-1 GP equation reduces to the DM equation (33) with $\mathcal{C}_{I}=c_{0}$ and $\rho_{0}(X)=\rho_{D M}(X)$ of Eq. (34). From Eqs. 45)- (46), the simple criterion for the validity of TFA in this case is $N\left(a_{0}+2 a_{2}\right) /\left(3 l_{0}\right)>>1$, which is consistent with the fact that for $m=0, c_{1}$ term does not contribute to the energy of the system.

The TF analysis shows that the spatial extents of the components are different for an anti-ferromagnetic BEC for any non-zero magnetization, viz. Eqs. (43)-(44), which is also manifested by different chemical potentials, viz. Eqs. (38) and (40), whereas these are the same for a ferromagnetic BEC. Thus, in the domain $l_{\mathcal{D}(-1)} \leq X<l_{\mathcal{D}(+1)}$ only component $m_{f}=+1$ survives for the anti-ferromagnetic BEC effectively separating this component from mixed phase in the $X<l_{\mathcal{D}(-1)}$ domain. The different spatial extents of the components for an anti-ferromagnetic BEC also imply that SMA is not valid in general except for $m=0$, when $l_{\mathcal{D}( \pm 1)}$ of Eqs. 43 (44) become equal. The ground states shown in Figs. 3 and 4 preserve the symmetry of the trapping potential. These symmetric profiles minimize $c_{1}$-dependent interaction energy 30

$$
\begin{aligned}
E_{\mathrm{A}}= & \frac{c_{1}}{2} \int\left[\left(\rho_{+1}-\rho_{-1}\right)^{2}+2 \rho_{0}\left(\rho_{+1}+\rho_{-1}\right)\right. \\
& \left.-4 \sqrt{\rho_{+1} \rho_{-1}} \rho_{0}\right] d \mathbf{x} .
\end{aligned}
$$

The asymmetric states, where the two phase-separated components lie side by side [13], do not minimize $E_{\mathrm{A}}$ in addition to having more potential-energy contribution. Hence, they do not emerge as the ground states in trapped spinor condensates. The asymmetric states can emerge as the ground state in the presence of Zeeman energy [2, 11] or spin-orbit coupling [13, 15] which we do not include in the Hamiltonian.

\section{ANALYTIC MODEL FOR SPIN-2 BEC}

\section{A. Ferromagnetic BEC}

In this case, the component densities are given by the DM equation (23) along with distributions (24)-26) and the analytic model is derived from the TFA to the DM equation 13. Following the procedure of Sec. III A for a spin-1 ferromagnetic BEC, the TFA densities are again given by Eq. (34), but now with $C \equiv \mathcal{C}_{I I}=c_{0}+4 c_{1}$. The component densities are then obtained using Eqs. (24)-(26). These analytic densities for a quasi-1D spin-2 ferromagnetic ${ }^{23} \mathrm{Na}$ and ${ }^{83} \mathrm{Rb}$ BECs are shown in Figs. 5 (a) and (b), respectively, together with the numerical densities from the full coupled GP equations (17)- 190 .

In the DM model $\mathcal{C}_{I I} \sim a_{4}$ plays the same role as the scattering length $a$ in a scalar BEC in Eq. (1). Hence in this case the condition of validity of the TFA will be $N a_{4} / l_{0}>>1$.

\section{B. Anti-ferromagnetic BEC}

The analytic model here is obtained from the TFA to the GP equation (17) involving only $\phi_{ \pm 2}(\mathbf{x})$ subject to $\phi_{0}(\mathbf{x})=\phi_{ \pm 1}(\mathbf{x})=0$ with normalization condition 30 . After neglecting the kinetic energy terms in the GP equation (17) the corresponding TFA densities are given by

$$
\mu_{ \pm 2}=X^{2} / 2+c_{0} \rho \pm 4 c_{1}\left(\rho_{+2}-\rho_{-2}\right)+\frac{2 c_{2} \rho_{\mp 2}}{5},
$$

For a non-zero magnetization $0<m \leq 2$, the $m_{f}=+2$ component extends to a larger domain $\left(X<l_{\mathcal{D}(+2)}\right)$ than the $m_{f}=-2$ component with a smaller extension $\left(X<l_{\mathcal{D}(-2)}, l_{\mathcal{D}(+2)}>l_{\mathcal{D}(-2)}\right)$. Following the procedure presented in Sec. IIIB for a spin-1 anti-ferromagnetic 

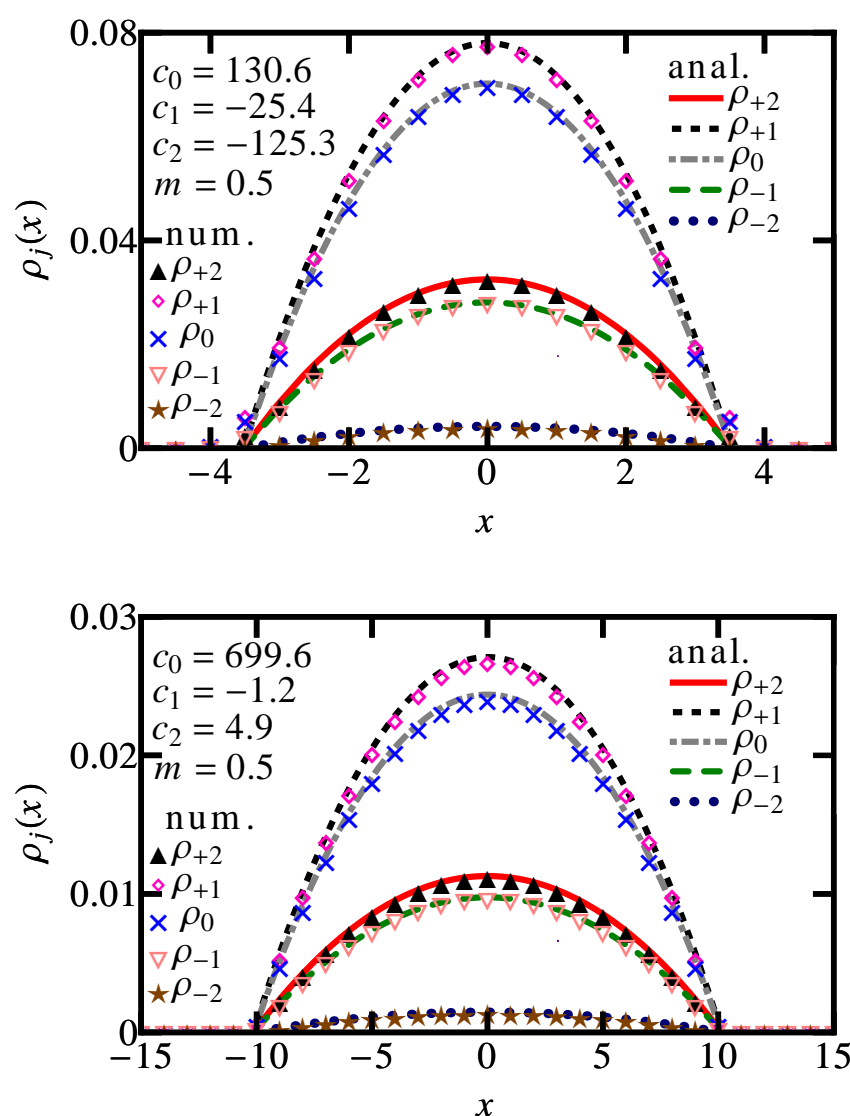

FIG. 5: (Color online) (a) and (b) show analytic (anal.) and numerical (num.) densities of quasi-1D spin-2 ferromagnetic ${ }^{23} \mathrm{Na}$ and ${ }^{83} \mathrm{Rb} \mathrm{BECs}$, respectively. For ${ }^{23} \mathrm{Na}$, the number of atoms, scattering lengths and oscillator lengths are, respectively, $N=10,000, a_{0}=34.9 a_{B}, a_{2}=45.8 a_{B}, a_{4}=6.45 a_{B}$, [6] $l_{0}=4.69 \mu \mathrm{m}, l_{y z}=1.05 \mu \mathrm{m}$, here $a_{B}$ is Bohr radius. The experimental value of $a_{4}\left(=64.5 a_{B}\right)$ has been modified to access the ferromagnetic phase of ${ }^{23} \mathrm{Na}$ (using a Feshbach resonance) from the natural anti-ferromagnetic phase. For ${ }^{83} \mathrm{Rb}$, the corresponding parameters are $N=10,000, a_{0}=83.0 a_{B}$, $a_{2}=82.0 a_{B}, a_{4}=81.0 a_{B}$, [6] $l_{0}=2.47 \mu \mathrm{m}, l_{y z}=0.55 \mu \mathrm{m}$.

BEC, one can calculate $l_{\mathcal{D}(+2)}$ in $1 \mathrm{D}, 2 \mathrm{D}$ and $3 \mathrm{D}$, respectively, as

$l_{1(+2)}=\frac{[3 \mathcal{A}]^{1 / 3}}{20^{1 / 3}}, \quad l_{2(+2)}=\frac{[2 \mathcal{A}]^{1 / 4}}{(5 \pi)^{1 / 4}}, \quad l_{3(+2)}=\frac{[3 \mathcal{A}]^{1 / 5}}{(8 \pi)^{1 / 5}}$,

where $\mathcal{A}=\left(10 c_{0}+2 c_{2}+20 c_{1} m-c_{2} m\right)$. Similarly, $l_{\mathcal{D}(-2)}$ in $1 \mathrm{D}, 2 \mathrm{D}$ and $3 \mathrm{D}$, respectively, are

$l_{1(-2)}=\frac{[3 \mathcal{B}]^{1 / 3}}{20^{1 / 3}}, \quad l_{2(-2)}=\frac{[2 \mathcal{B}]^{1 / 4}}{(5 \pi)^{1 / 4}}, \quad l_{3(-2)}=\frac{[3 \mathcal{B}]^{1 / 5}}{(8 \pi)^{1 / 5}}$,

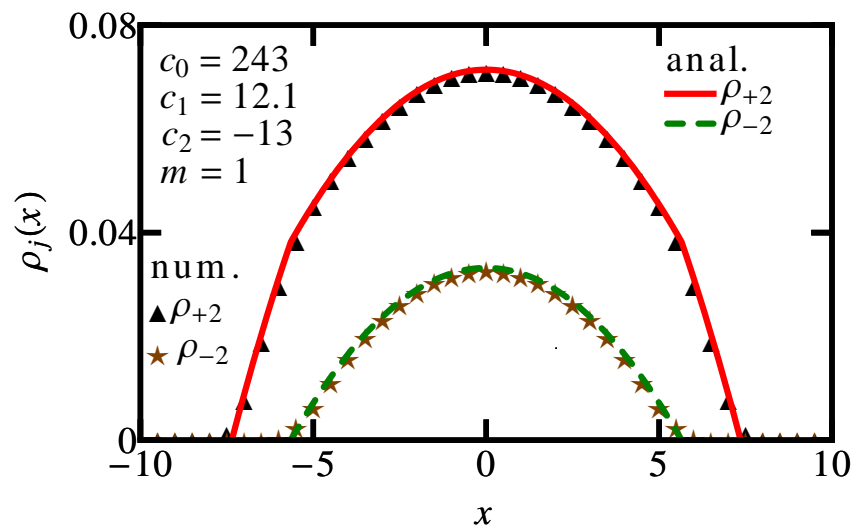

FIG. 6: (Color online) Analytic (anal.) and numerical (num.) densities of a spin-2 quasi-1D anti-ferromagnetic ${ }^{23} \mathrm{Na}$ BEC. The number of atoms, scattering lengths and oscillator lengths are, respectively, $N=10,000 ; a_{0}=34.9 a_{B}$, $a_{2}=45.8 a_{B}, a_{4}=64.5 a_{B}$ [6] $; l_{0}=4.69 \mu \mathrm{m}, l_{y z}=1.05 \mu \mathrm{m}$.

where $\mathcal{B}=\left(5 c_{0}+c_{2}\right)(2-m)$. The analytic TFA densities of the spin components $m_{f}= \pm 2$ are given by

$$
\begin{aligned}
\rho_{+2}(X) & =\frac{20 c_{1}\left(l_{\mathcal{D}(-2)}^{2}-X^{2}\right)+2 c_{2}\left(l_{\mathcal{D}(+2)}^{2}-l_{\mathcal{D}(-2)}^{2}\right)}{4 \mathcal{C}_{I I}\left(5 c_{0}+c_{2}\right)} \\
+ & \frac{5 c_{0}\left(2 l_{\mathcal{D}(+2)}^{2}-l_{\mathcal{D}(-2)}^{2}-X^{2}\right)}{4 \mathcal{C}_{I I}\left(5 c_{0}+c_{2}\right)}, \quad X \leq l_{\mathcal{D}(-2)} \\
\rho_{+2}(X) & =\frac{l_{\mathcal{D}(+2)}^{2}-X^{2}}{2 \mathcal{C}_{I I}}, \quad l_{\mathcal{D}(+2)} \geq X \geq l_{\mathcal{D}(-2)} \\
\rho_{-2}(X) & =\frac{5\left(l_{\mathcal{D}(-2)}^{2}-X^{2}\right)}{4\left(5 c_{0}+c_{2}\right)}, \quad X \leq l_{\mathcal{D}(-2)}
\end{aligned}
$$

with the extensions $l_{ \pm 2}$ given by Eqs. (49) and (50). The analytic and numerical densities for a quasi-1D spin-2 anti-ferromagnetic ${ }^{23} \mathrm{Na}$ BEC are compared in Fig. 6 . Again, in this case, phase-separated asymmetric profiles do not emerge as ground states due to more energy contribution from $c_{2}$-dependent energy term in addition to more potential energy as compared to symmetric profiles.

Comparing Eq. (1) with Eqs. (49)-(50), the conditions for the validity of TFA in this case are

$$
\begin{aligned}
\frac{N\left[c_{0 a}+2 c_{1 a} m+c_{2 a}(2-m) / 10\right]}{7 l_{0}} & >1, \\
\frac{N\left[\left(c_{0 a}+c_{2 a} / 5\right)(1-m / 2)\right]}{7 l_{0}} & >>1,
\end{aligned}
$$

for $m_{f}=+2$ and $m_{f}=-2$ components, respectively, where $c_{0 a}=4 a_{2}+3 a_{4}, c_{1 a}=a_{4}-a_{2}$, and $c_{2 a}=7 a_{0}-$ $10 a_{2}+3 a_{4}$.

For $m=0$ there is another degenerate ground state with the all the atoms in the $m_{f}=0$ component [3]. In 
this case the GP equation reduces to the DM equation (33) with $\mathcal{C}_{I I}=\left(c_{0}+c_{2} / 5\right)$ and $\rho_{0}(X)=\rho_{D M}(X)$ of (34). A superposition of this solution and the solution corresponding to Eq. (48) with $m=0$ will also be a degenerate solution. The simpler criterion for the validity of TFA in this case is $N\left[\left(7 a_{0}+10 a_{2}+18 a_{4}\right) / 5\right] /\left(7 l_{0}\right)>>$ 1 , which is consistent with Eqs. (54) and with $m=$ 0 .

\section{Cyclic BEC}

In this case, there are two degenerate ground states for all magnetization $m$ with non-zero component densities given by Eqs. (31) and (32), respectively. The analytic models will be obtained in these two cases from the TFA to the GP equations (17)- 19.

The former distribution (31) involves only two non-zero components in the GP equation. After neglecting the kinetic energy terms in the GP equations (17)-(19), the TFA densities for the non-zero spin components $m_{f}=+2$ and $m_{f}=-1$ are described by

$$
\begin{gathered}
\mu_{+2}=X^{2} / 2+c_{0} \rho+2 c_{1}\left(2 \rho_{+2}-\rho_{-1}\right), \\
\mu_{-1}=X^{2} / 2+c_{0} \rho-c_{1}\left(2 \rho_{+2}-\rho_{-1}\right) .
\end{gathered}
$$

For a non-zero magnetization $(2>m>0)$ the $m_{f}=+2$ component has a larger spatial extension $\left(l_{\mathcal{D}(+2)}\right)$ than the $m_{f}=-1$ component with a smaller spatial extension $\left( \pm l_{\mathcal{D}(-1)}, l_{\mathcal{D}(+2)}>l_{\mathcal{D}(-1)}\right)$. Following the procedure discussed for a spin-1 anti-ferromagnetic BEC in Sec. IIIB.

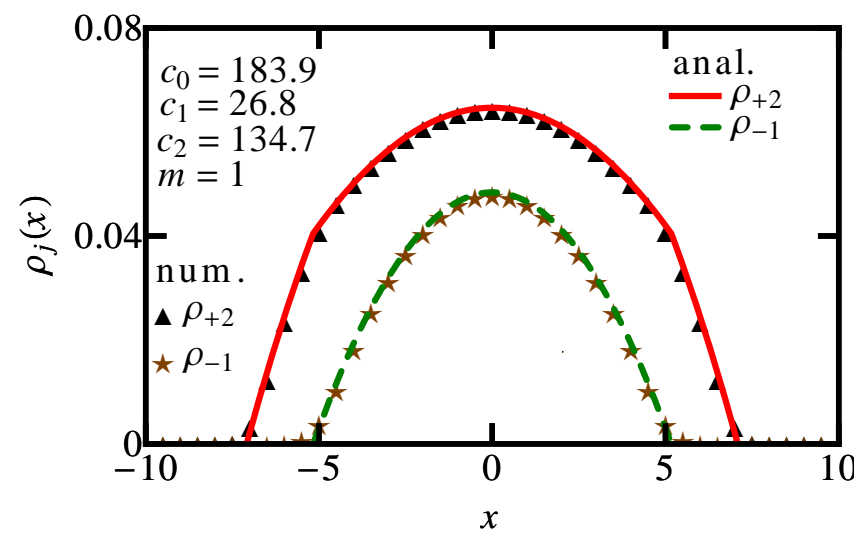

FIG. 7: (Color online) Analytic (anal.) and numerical (num.) densities of a spin-2 quasi-1D cyclic ${ }^{23} \mathrm{Na}$ BEC. The number of atoms, scattering lengths and oscillator lengths are, respectively, $N=10,000, a_{0}=34.9 a_{B}, a_{2}=22.9 a_{B}, a_{4}=64.5 a_{B}$ [6, $l_{0}=4.69 \mu \mathrm{m}, l_{y z}=1.05 \mu \mathrm{m}$. The experimental value of $a_{2}\left(=45.8 a_{B}\right)$ has been modified to access the cyclic phase of ${ }^{23} \mathrm{Na}$ (using a Feshbach resonance) from its natural antiferromagnetic phase.

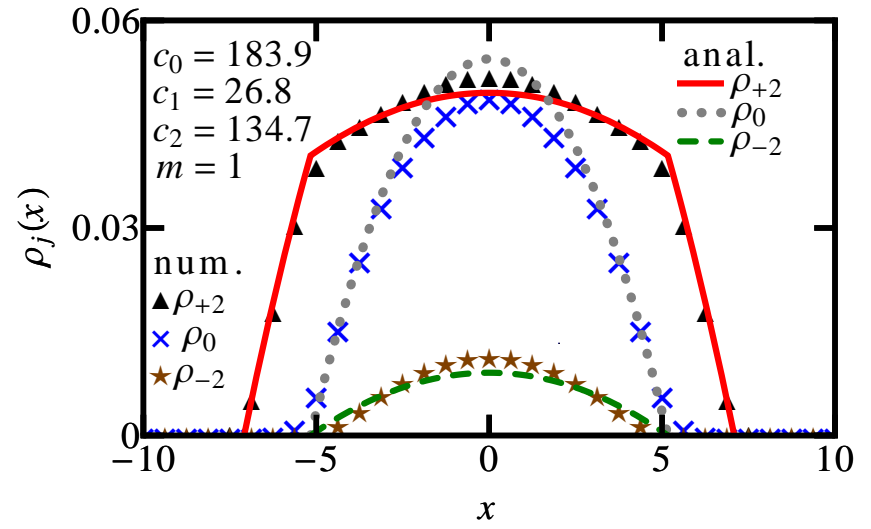

FIG. 8: (Color online) Analytic (anal.) and numerical (num.) densities of a spin-2 quasi-1D ${ }^{23} \mathrm{Na}$ cyclic BEC. All parameters are the same as in Fig. 7.

one obtains

$$
\begin{aligned}
& l_{\mathcal{D}(+2)}=l_{\mathcal{D}}\left[\left(c_{0}+2 c_{1} m\right) / \mathcal{C}_{I}\right]^{1 /(2+\mathcal{D})}, \\
& l_{\mathcal{D}(-1)}=l_{\mathcal{D}}\left[c_{0}(2-m) / 2 \mathcal{C}_{I}\right]^{1 /(2+\mathcal{D})}
\end{aligned}
$$

The normalized densities are given by

$\rho_{-1}(X)=\frac{\left(l_{\mathcal{D}(-1)}^{2}-X^{2}\right)}{3 c_{0}}, \quad X \leq l_{\mathcal{D}(-1)}$,
$\rho_{+2}(X)=\frac{3 c_{0} l_{\mathcal{D}(+2)}^{2}-2\left(c_{0}-2 c_{1}\right) l_{\mathcal{D}(-1)}^{2}-\mathcal{C}_{I I} X^{2}}{6 c_{0} \mathcal{C}_{I I}}, X \leq l_{\mathcal{D}(-1)}$

$$
=\frac{l_{\mathcal{D}(+2)}^{2}-X^{2}}{2 \mathcal{C}_{I I}} . \quad l_{\mathcal{D}(-1)} \leq X \leq l_{\mathcal{D}(+2)},
$$

Equations 60 - 62 together with extensions given by Eqs. (58)-(59) are the analytic densities in this case. The analytic and numerical densities for a quasi-1D spin2 cyclic ${ }^{23} \mathrm{Na}$ BEC are shown in Fig. 7. For $m=0$, SMA becomes exact for the cyclic phase of spin-2 condensate, as the $c_{1}$ and $c_{2}$-dependent terms in Eqs. (56)-(57) vanish.

Comparing Eq. (1) and Eqs. (58)-(59), the conditions for the validity of TFA in this case are

$$
\begin{aligned}
\frac{N\left[4 a_{2}+3 a_{4}+2\left(a_{4}-a_{2}\right) m\right]}{7 l_{0}} & >1, \\
\frac{N\left[4 a_{2}+3 a_{4}(1-m / 2)\right]}{7 l_{0}} & >>1,
\end{aligned}
$$

for $m_{f}=+2$ and $m_{f}=-1$ components, respectively. For $m=0$ the simple criterion for the validity of TFA is $N\left(4 a_{2}+3 a_{4}\right) /\left(7 l_{0}\right)>>1$, which is consistent with fact that only spin-independent non-linearity $\left(c_{0}\right.$ dependent term) contributes to the energy of the system.

Similarly, for the latter distribution (32), after neglecting the kinetic energy terms in the GP equations (17)- 
19, the TFA densities are given by

$$
\begin{aligned}
\mu_{ \pm 2} & =X^{2} / 2+c_{0} \rho \pm 4 c_{1}\left(\rho_{+2}-\rho_{-2}\right), \\
\mu_{0} & =X^{2} / 2+c_{0} \rho .
\end{aligned}
$$

This set of equations for $\rho_{ \pm 2}$ and $\rho_{0}$ is overcomplete and does not determine the densities. However, if we assume, consistent with Eq. 32, that

$$
\rho_{0}=\frac{2(2+m) \rho_{-2}}{2-m},
$$

then we can solve Eqs. 65 for $\rho_{ \pm 2}$ and obtain $\rho_{0}$ from Eq. (67). For $2>m>0$ the spatial extent $\left(l_{\mathcal{D}(+2)}\right)$ of density $\rho_{+2}$ is larger than the spatial extent $\left(l_{\mathcal{D}(-2)}\right)$ of density $\rho_{-2}$. Equations 65 can then be be solved to obtain

$$
\begin{aligned}
\rho_{+2}(X) & =\frac{4 c_{1}(m-2)\left(X^{2}-\delta\right)+c_{0} \kappa(6+m)}{64 c_{0} c_{1}}, \quad X \leq l_{\mathcal{D}(-2)} \\
& =\frac{2 \mu_{+2}-X^{2}}{2 \mathcal{C}_{I I}}, \quad l_{\mathcal{D}(+2) \geq X \geq l_{\mathcal{D}(-2)},} \\
\rho_{-2}(X) & =\frac{(m-2)\left[c_{0} \kappa+4 c_{1}\left\{X^{2}-\delta\right\}\right]}{64 c_{0} c_{1}}, \quad X \leq l_{\mathcal{D}(-2)},
\end{aligned}
$$

where $\delta=\mu_{+2}+\mu_{-2}, \kappa=\mu_{+2}-\mu_{-2}$. with the chemical potentials $\mu_{+2}$ and $\mu_{-2}$ given by

$$
\mu_{+2}=\frac{l_{\mathcal{D}(+2)}^{2}}{2}, \quad \mu_{-2}=\frac{\left(c_{0}-4 c_{1}\right) l_{\mathcal{D}(+2)}^{2}+8 c_{1} l_{\mathcal{D}(-2)}^{2}}{2 \mathcal{C}_{I I}}
$$

where $l_{\mathcal{D}(+2)}$ and $l_{\mathcal{D}(-2)}$ are the same as $l_{\mathcal{D}(+2)}$ and $l_{\mathcal{D}(-1)}$ of Eqs. (58)- (59), respectively. After substituting the expressions for chemical potentials $\mu_{ \pm 2}$ given by Eqs. (71) in Eqs. 67)- 70 , we obtain the final densities $\rho_{ \pm 2}$ and $\rho_{0}$ as

$$
\begin{aligned}
& \rho_{+2}(X)=\frac{c_{0}\left[8 l_{\mathcal{D}(+2)}^{2}-l_{\mathcal{D}(-2)}^{2}(6+m)+(-2+m) X^{2}\right]}{16 c_{0} \mathcal{C}_{I I}} \\
&+ \frac{4 c_{1}(2-m)\left(l_{\mathcal{D}(-2)}^{2}-X^{2}\right)}{16 c_{0} \mathcal{C}_{I I}}, \quad X \leq l_{\mathcal{D}(-2)}, \quad(72) \\
& \rho_{+2}(X)= \frac{l_{\mathcal{D}(+2)}^{2}-X^{2}}{2 \mathcal{C}_{I I}}, \quad l_{\mathcal{D}(+2)} \geq X \geq l_{\mathcal{D}(-2)}, \quad(73) \\
& \rho_{0}(X)=\frac{2(2+m) \rho_{-2}}{2-m}, \quad X \leq l_{\mathcal{D}(-2)}, \\
& \rho_{-2}(X)=\frac{(2-m)\left(l_{\mathcal{D}(-2)}^{2}-X^{2}\right)}{16 c_{0}}, \quad X \leq l_{\mathcal{D}(-2)}(75) \\
& \rho_{0}(X)=\rho_{-2}=0, \quad l_{\mathcal{D}(+2)} \geq X \geq l_{\mathcal{D}(-2)} . \quad(76)
\end{aligned}
$$

The analytic and numerical densities in this case for a spin-2 quasi-1D ${ }^{23} \mathrm{Na}$ cyclic BEC are shown in Fig. 8 .
The criteria for the validity of TFA in this case are again given by Eqs. (63)- 64) for $m_{f}=+2$ and $m_{f}=-2$ components, receptively. Thus, for a spin- $2{ }^{23} \mathrm{Na}$ cyclic $\mathrm{BEC}$, there are two distinct degenerate ground states as are shown in Figs. 7 and 8. The Hamiltonian of the spinor BEC is time-reversal invariant, yet the degenerate states shown in Figs. 7 and 8 break time reversal symmetry. Time-reversal symmetry-breaking states in spinor BECs were previously studied [15]. In cyclic phase too the additional potential energy cost rules out the possibility of asymmetric phase-separated profiles as ground states.

\section{CONCLUDING REMARKS}

The mean-field GP equation for a spin-1 and spin2 spinor BEC involve three- and five-component complex wave function. Some simplification emerges for the ground-state wave function of a spinor BEC. For an antiferromagnetic or cyclic BEC with a non-zero magnetization, some of the spin-component wave functions become zero, thus reducing the original GP equation with three or five components to a system of coupled equations with only two or three components, which we call a reduced GP equation. For a ferromagnetic BEC with a non-zero magnetization the densities of different spin components for the ground-state wave function are found to be multiples of each other. This allows to solve the density according to a single GP equation, which we call the decoupled-mode (DM) equation, and calculate the densities of different spin components as multiples of a single DM density. These reduced GP and DM equations are valid in all spatial dimensions. Here we suggest simple analytic models for the ground-state densities of a spinor BEC obtained by applying Thomas-Fermi approximation to the DM and reduced GP equations. These analytic results for densities are found to be in good agreement with those obtained from the numerical solution of the full GP equation for ferromagnetic, anti-ferromagnetic, and cyclic spin-1 and spin-2 spinor BECs. Although, we considered in this paper nearly-overlapping configurations of the spinor components, the presence of Zeeman energy and spin-orbit coupling in the Hamiltonian can lead to asymmetric phase-separated configurations [13, 15] as ground states. An investigation leading to the analytic densities of the phase-separated solutions would be an interesting future work.

\section{Acknowledgments}

This work is financed by the Fundação de Amparo à Pesquisa do Estado de São Paulo (Brazil) under Contract Nos. 2013/07213-0, 2012/00451-0 and also by the Conselho Nacional de Desenvolvimento Científico e Tecnológico (Brazil). 


\section{APPENDIX A}

\section{A. Ferromagnetic spin-1 BEC}

For the ground state of a spin-1 ferromagnetic BEC $\left(c_{1}<0\right)$, the coefficients $\alpha_{j}$, can be obtained from a minimization of the energy

$$
E=\frac{N}{2} \int\left[\sum_{j=-1}^{1}\left|\phi_{j}^{\prime}\right|^{2}+x^{2} \rho+c_{0} \rho^{2}+c_{1}|\mathbf{F}|^{2}\right] d \mathbf{x},
$$

where prime denotes $x$ derivative. Assuming that component wave functions are given by the DM ansatz (12), to minimize energy $E$ we need to maximize the positive integral $\int|\mathbf{F}|^{2} d \mathbf{x}$

$$
\begin{aligned}
\int|\mathbf{F}|^{2} d \mathbf{x} & =\left[2\left|\left(\alpha_{+1}^{*} \alpha_{0}+\alpha_{0}^{*} \alpha_{-1}\right)\right|^{2}+m^{2}\right] \mathcal{I}, \\
\mathcal{I} & =\int \phi_{\mathrm{DM}}^{4}(x) d \mathbf{x} .
\end{aligned}
$$

Now, writing $\alpha_{j}=\left|\alpha_{j}\right| e^{i \theta_{j}}$, we get

$$
\begin{aligned}
\int|\mathbf{F}|^{2} d \mathbf{x}= & {\left[2|| \alpha_{+1}|| \alpha_{0}|+| \alpha_{0}|| \alpha_{-1}\left|e^{i\left(\theta_{+1}+\theta_{-1}-2 \theta_{0}\right)}\right|^{2}\right.} \\
& \left.+m^{2}\right] \mathcal{I}
\end{aligned}
$$

To maximize integral 80 , we take $\exp \left(\theta_{+1}+\theta_{-1}-2 \theta_{0}\right)=$ 1 and obtain

$$
\int|\mathbf{F}|^{2} d \mathbf{x}=\left[2\left(\left|\alpha_{+1}\right|\left|\alpha_{0}\right|+\left|\alpha_{0}\right|\left|\alpha_{-1}\right|\right)^{2}+m^{2}\right] \mathcal{I} .
$$

For a fixed magnetization $m$ and DM function $\phi_{\mathrm{DM}}$, the maximization of $\int|\mathbf{F}|^{2} d \mathbf{x}$ corresponds to finding the stationary points of the following "Lagrange" function

$$
\begin{aligned}
& L_{1}\left(\left|\alpha_{j}\right|, \lambda_{1}, \lambda_{2}\right)=2\left(\left|\alpha_{+1}\right|\left|\alpha_{0}\right|+\left|\alpha_{0}\right|\left|\alpha_{-1}\right|\right)^{2} \\
& +\lambda_{1}\left(1-\sum_{j}\left|\alpha_{j}\right|^{2}\right)+\lambda_{2}\left(m-\left|\alpha_{+1}\right|^{2}+\left|\alpha_{-1}\right|^{2}\right) .
\end{aligned}
$$

Here $\lambda_{1}$ and $\lambda_{2}$ are Lagrangian multipliers to fix the normalization and magnetization to 1 and $m$, respectively. The stationary points of $L_{1}$ are determined by the following Lagrange equations

$$
\frac{\partial L_{1}}{\partial\left|\alpha_{j}\right|}=0, \quad \frac{\partial L_{1}}{\partial \lambda_{1}}=0, \quad \frac{\partial L_{1}}{\partial \lambda_{2}}=0 .
$$

with solution (14) together with $\lambda_{1}=2, \lambda_{2}=-2 \mathrm{~m}$.

\section{B. Anti-ferromagnetic spin-1 BEC}

In case of an anti-ferromagnetic BEC $\left(c_{1}>0\right)$, $\int|\mathbf{F}|^{2} d \mathbf{x}=\int\left(F_{+} F_{-}+F_{z}^{2}\right) d \mathbf{x}$ is minimized by making $\phi_{0}(x)=0$ for any $m \neq 0$ and the densities satisfy Eq.
(16). If we further assume the DM ansatz (12), the coefficients $\alpha_{j}$ can be obtained from a minimization of $(80)$. For this, we take $\exp \left(\theta_{+1}+\theta_{-1}-2 \theta_{0}\right)=-1$ and obtain

$$
\int|\mathbf{F}|^{2} d \mathbf{x}=\left[2\left(\left|\alpha_{+1}\right|\left|\alpha_{0}\right|-\left|\alpha_{0}\right|\left|\alpha_{-1}\right|\right)^{2}+m^{2}\right] \mathcal{I}
$$

Following the procedure discussed for a ferromagnetic BEC, one can minimize $\int|\mathbf{F}|^{2} d \mathbf{x}$ under the twin constraints of fixed norm and magnetization and, in agreement with Eq. 16), obtain

$$
\left|\alpha_{ \pm 1}\right|=\sqrt{\frac{1 \pm m}{2}}, \quad \alpha_{0}=0 .
$$

\section{APPENDIX B}

\section{A. Ferromagnetic spin-2 BEC}

For a spin-2 ferromagnetic BEC $\left(c_{1}<0, c_{2}>20 c_{1}\right)$, the energy is given by

$E=\frac{N}{2} \int\left[\sum_{j=-2}^{2}\left|\phi_{j}^{\prime}\right|^{2}+x^{2} \rho+c_{0} \rho^{2}+c_{1}|\mathbf{F}|^{2}+c_{2}|\Theta|^{2}\right] d \mathbf{x}$.

For a ferromagnetic BEC, the energy minimization corresponds to a maximization of the $c_{1}$-dependent term $\int|\mathbf{F}|^{2} d \mathbf{x}$. We find that this automatically minimizes the $c_{2}$-dependent term $\int|\Theta|^{2} d \mathbf{x}$ to zero. Assuming the DM ansatz (23) we seek the coefficients $\alpha_{j}$ which maximize $\int|\mathbf{F}|^{2} d \mathbf{x}$. Following the procedure for ferromagnetic spin-1 BEC, we can write

$$
\begin{aligned}
& \int|\mathbf{F}|^{2} d \mathbf{x}=\left[\mid 2\left\{\left|\alpha_{+2}\right|\left|\alpha_{+1}\right|+\left|\alpha_{-2}\right|\left|\alpha_{-1}\right|\right.\right. \\
& \left.e^{i\left(\theta_{-2}-\theta_{-1}-\theta_{1}+\theta_{2}\right)}\right\}+\sqrt{6} e^{i\left(\theta_{0}-2 \theta_{1}+\theta_{2}\right)} \\
& \left.\left.\left\{\left|\alpha_{+1}\right|\left|\alpha_{0}\right|+\left|\alpha_{0}\right|\left|\alpha_{-1}\right| e^{i\left(\theta_{-1}-2 \theta_{0}+\theta_{1}\right)}\right\}\right|^{2}+m^{2}\right] \mathcal{I} .
\end{aligned}
$$

To maximize 86 we take all exponential factors in this equation to be +1 and obtain

$$
\begin{aligned}
\int|\mathbf{F}|^{2} d \mathbf{x} & =\left[\left\{2\left(\left|\alpha_{+2}\right|\left|\alpha_{+1}\right|+\left|\alpha_{-2}\right|\left|\alpha_{-1}\right|\right)\right.\right. \\
& \left.\left.+\sqrt{6}\left(\left|\alpha_{+1}\right|\left|\alpha_{0}\right|+\left|\alpha_{0}\right|\left|\alpha_{-1}\right|\right)\right\}^{2}+m^{2}\right] \mathcal{I}
\end{aligned}
$$

For a fixed $m(2>m>0)$ and $\mathcal{I}$, the maximization of $\int|\mathbf{F}|^{2} d \mathbf{x}$ corresponds to finding the stationary points of the following Lagrange function

$$
\begin{aligned}
L_{2}\left(\left|\alpha_{j}\right|, \lambda_{1}, \lambda_{2}\right) & =2\left(\left|\alpha_{+2}\right|\left|\alpha_{+1}\right|+\left|\alpha_{-2}\right|\left|\alpha_{-1}\right|\right)+\sqrt{6}\left|\alpha_{0}\right| \\
& \left(\left|\alpha_{+1}\right|+\left|\alpha_{-1}\right|\right)+\lambda_{1}\left(1-\sum_{j}\left|\alpha_{j}\right|^{2}\right)+\lambda_{2} \\
& \left(m-2\left|\alpha_{+2}\right|^{2}-\left|\alpha_{+1}\right|^{2}+\left|\alpha_{-1}\right|^{2}+2\left|\alpha_{-2}\right|^{2}\right) .
\end{aligned}
$$


Here $\lambda_{1}$ and $\lambda_{2}$ have the same meaning as in Eq. 82 . The stationary point which maximizes $\int|\mathbf{F}|^{2} d \mathbf{x}$ thus yields Eqs. 24 -26 together with

$$
\lambda_{1}=\frac{4}{\sqrt{\left(4-m^{2}\right)}}, \quad \lambda_{2}=-\frac{m}{\sqrt{\left(4-m^{2}\right)}} .
$$

Using Eqs. (24)-(26), we find that these $\alpha_{j}$ 's also minimize $\int|\Theta|^{2} d \mathbf{x}$ to 0 which guarantees that the state so obtained is the ground state.

\section{B. Anti-ferromagnetic spin-2 BEC}

Similarly in anti-ferromagnetic subdomain $\left(c_{2}<0\right.$ and $\left.c_{2}<20 c_{1}\right)$, the energy minimization corresponds to a maximization of the $c_{2}$-dependent term $\int|\Theta|^{2} d \mathbf{x}$. Assuming DM ansatz 16 and $\alpha_{j}=\left|\alpha_{j}\right| \exp \left(i \theta_{j}\right)$ we have

$$
\begin{aligned}
\int|\Theta|^{2} d \mathbf{x}= & |2| \alpha_{+2}|| \alpha_{-2}|-2| \alpha_{+1}|| \alpha_{-1} \mid e^{i\left(\theta_{1}+\theta_{-1}-\theta_{2}-\theta_{-2}\right)} \\
& +\left.\left|\alpha_{0}\right|^{2} e^{i\left(2 \theta_{0}-\theta_{2}-\theta_{-2}\right)}\right|^{2} \mathcal{I}
\end{aligned}
$$

To maximize integral 89 we take the first exponential to be -1 and the second exponential to be +1 . For a fixed $m(2>m>0)$ and $\mathcal{I}$, the maximization of $\int|\Theta|^{2} d \mathbf{x}$ corresponds to finding the stationary points of the following Lagrange function

$$
\begin{aligned}
L_{\theta}\left(\left|\alpha_{j}\right|, \lambda_{1}, \lambda_{2}\right)= & \left(2\left|\alpha_{+2}\right|\left|\alpha_{-2}\right|+2\left|\alpha_{+1}\right|\left|\alpha_{-1}\right|+\left|\alpha_{0}\right|^{2}\right) \\
& +\lambda_{1}\left(1-\sum_{j}\left|\alpha_{j}\right|^{2}\right)+\lambda_{2}\left(m-2\left|\alpha_{+2}\right|^{2}\right. \\
& \left.-\left|\alpha_{+1}\right|^{2}+\left|\alpha_{-1}\right|^{2}+2\left|\alpha_{-2}\right|^{2}\right) .
\end{aligned}
$$

The stationary point, which maximizes $\int|\Theta|^{2} d \mathbf{x}$, yields

$$
\begin{aligned}
\left|\alpha_{ \pm 2}\right| & =\frac{\sqrt{2 \pm m}}{2}, \quad \alpha_{ \pm 1}=\alpha_{0}=0 \\
\lambda_{1} & =\frac{2}{\sqrt{\left(4-m^{2}\right)}}, \quad \lambda_{2}=\frac{-m}{2 \sqrt{\left(4-m^{2}\right)}} .
\end{aligned}
$$

Using Eqs. (91)- 92 , we find that $\int|\mathbf{F}|^{2} d \mathbf{x}$ has the minimum value $m^{2} \mathcal{I}$ which guarantees that the state so obtained is the ground state.

\section{Cyclic spin-2 BEC}

For a spin-2 cyclic BEC $c_{1}>0$ and $c_{2}>0$, energy minimization involves minimization of both $\int|\mathbf{F}|^{2} d \mathbf{x}$ and $\int|\Theta|^{2} d \mathbf{x}$ to their respective minimum values $m^{2} \mathcal{I}$ and 0 , respectively. From equations 86 and 89 , one can see that for $0<m<2$, consistent with Eqs. (31)- 32 , there are only two possibilities for the ground states:

$$
(i)\left|\alpha_{ \pm 2}\right|=\frac{2 \pm m}{4}, \quad\left|\alpha_{0}\right|=\frac{\sqrt{4-m^{2}}}{\sqrt{8}}, \quad \alpha_{ \pm 1}=0,
$$
provided that $\exp \left(2 \theta_{0}-\theta_{+2}-\theta_{-2}\right)=-1$, and

$(i)\left|\alpha_{+2}\right|=\frac{\sqrt{1+m}}{\sqrt{3}}, \quad\left|\alpha_{-1}\right|=\frac{\sqrt{2-m}}{\sqrt{3}}, \quad \alpha_{ \pm 1}=\alpha_{0}=0$.
[1] D. M. Stamper-Kurn, M. R. Andrews, A. P. Chikkatur, S. Inouye, H.-J. Miesner, J. Stenger, and W. Ketterle, Phys. Rev. Lett. 802027 (1998).

[2] Y. Kawaguchi and M. Ueda, Phys. Rep. 520, 253 (2012).

[3] M. Ueda, Rep. Prog. Phys. 77, 122401 (2014).

[4] D. M. Stamper-Kurn and M. Ueda, Rev. Mod. Phys. 85, 1191 (2013).

[5] T. Ohmi and K. Machida, J. Phys. Soc. Japan 67, 1822 (1998); T.-L. Ho, Phys. Rev. Lett. 81742 (1998).

[6] C. V. Ciobanu, S.-K. Yip, and T.-L. Ho, Phys. Rev. A 61033607 (2000);

[7] M. Ueda and M. Koashi, Phys. Rev. A 65063602 (2002); H. Saito and M. Ueda, Phys. Rev. A 72053628 (2005).

[8] J. Stenger, S. Inouye, D.M. Stamper-Kurn, H.-J. Miesner, A.P. Chikkatur, and W. Ketterle, Nature 396, 345 (1998).

[9] D. R. Romano and E. J. V. de Passos, Phys. Rev. A 70, 043614 (2004); K. Murata, H. Saito, and M. Ueda, Phys. Rev. A 75013607 (2007).

[10] W. Zhang, S. Yi, and L. You, New J. Phys. 5, 77 (2003).

[11] M. Matuszewski, T. J. Alexander, and Y. S. Kivshar, Phys. Rev. A 80, 023602 (2009); M. Matuszewski, Phys. Rev. A 82, 053630 (2010).
[12] H. Wang, Int. J. of Computer Math. 84, 925 (2007); W. Bao and F. Y. Lim, Siam J. Sci. Comp. 30, 1925 (2008); F. Y. Lim and W. Bao, Phys. Rev. E 78, 066704 (2008).

[13] S. Gautam and S. K. Adhikari, Phys. Rev. A 90, 043619 (2014).

[14] H. Wang, J. Comput. Phys. 230, 6155 (2011); 274, 473 (2014).

[15] S. Gautam and S. K. Adhikari, Phys. Rev. A 91, 013624 (2015).

[16] L. Salasnich, A. Parola, and L. Reatto, Phys. Rev. A 65, 043614 (2002).

[17] G. Baym and C. J. Pethick, Phys. Rev. Lett. 76, 6 (1996).

[18] F. Dalfovo, L. Pitaevskii, and S. Stringari, J. Res. Natl. Stand. Technol. 101, 537 (1996); Rev. Mod. Phys. 71, 463 (1999).

[19] C. J. Pethick and H. Smith, Bose-Einstein condensation in dilute gases, Cambridge University Press, Cambridge (2002).

[20] P. Schuck and X. Viñas, Phys. Rev. A 61, 043603 (2000).

[21] T.-L. Ho and V. B. Shenoy, Phys. Rev. Lett. 77, 3276 (1996); M. Trippenbach, K. Góral, K. Rzazewski, B. Malomed, and Y. B. Band, J. Phys. B 33, 4017 (2000); S. T. Chui, V. N. Ryzhov, and E. E. Tareyeva, Phys. Rev. A 
63, 023605 (2001); JETP Lett. 75, 279 (2002); J. Polo, V. Ahufinger, P. Mason, S. Sridhar, T. P. Billam, and S. A. Gardiner, Phys. Rev. A 91, 053626 (2015); S. Gautam and D. Angom, J. Phys. B 43, 095302 (2010).

[22] J. Mur-Petit, Phys. Rev. A 79, 063603 (2009).

[23] A. Aftalion and P. Mason, Phys. Rev. A 88, 023610 (2013).

[24] E. G. M. Van kempen, S. J. J. M. F. Kokkelmans, D. J. Heinzen, and B. J. Verhaar, Phys. Rev. Lett. 88, 093201 (2002);

[25] M.-S. Chang, Q. Qin, W. Zhang, L. You, and M. S. Chapman, Nature Phys. 1, 111 (2005); A. Widera, F. Gerbier, S. Fölling, T. Gericke, O. Mandel, and I. Bloch, New J. Phys. 8, 152 (2006).

[26] A. T. Black, E. Gomez, L. D. Turner, S. Jung, and P. D. Lett, Phys. Rev. Lett. 99, 070403 (2007).
[27] S. Inouye, M. R. Andrews, J. Stenger, H.-J. Miesner, D. M. Stamper-Kurn, and W. Ketterle, Nature (London) 392, 151 (1998).

[28] C. Chin, R. Grimm, P. Julienne, and E. Tiesinga, Rev. Mod. Phys. 82, 1225 (2010).

[29] C. K. Law, H. Pu, and N. P. Bigelow, Phys. Rev. Lett. 81, 5257 (1998); H. Pu, C. K. Law, S. Raghavan, J. H. Eberly, and N. P. Bigelow, Phys. Rev. A 60, 1463 (1999).

[30] S. Yi, Ö. E. Müstecaplıŏ̆lu, C. P. Sun, and L. You, Phys. Rev. A 66, 011601(R) (2002).

[31] P. Muruganandam and S. K. Adhikari, Comput. Phys. Commun. 180, 1888 (2009); D. Vudragovic, I. Vidanovic, A. Balaz, P. Muruganandam, and S. K. Adhikari, Comput. Phys. Commun. 183, 2021 (2012). 